\title{
Overall Properties of a Discrete Membrane with Randomly Distributed Defects
}

\author{
Andrea Braides \& Andrey Piatnitski
}

\author{
Communicated by G. FRIESECKE
}

\begin{abstract}
A prototype for variational percolation problems with surface energies is considered: the description of the macroscopic properties of a (two-dimensional) discrete membrane with randomly distributed defects in the spirit of the weak membrane model of Blake and Zisserman (quadratic springs that may break at a critical length of the elongation). After introducing energies depending on suitable independent identically distributed random variables, this is done by exhibiting an equivalent continuum energy computed using $\Gamma$-convergence, geometric measure theory, and percolation arguments. We show that below a percolation threshold the effect of the defects is negligible and the continuum description is given by the Dirichlet integral, while above that threshold an additional (Griffith) fracture term appears in the energy, which depends only on the defect probability through the chemical distance on the "weak cluster of defects".
\end{abstract}

\section{Introduction}

In this paper we study the problem of the description of the overall behavior of a discrete membrane with defects. Our model mixes two extreme behaviors and is set in a variational framework. One extreme behavior is that of a "strong membrane", consisting just in the finite-difference approximation of the Dirichlet integral. The defects are introduced as nonlinear pair interactions as in BLAKE and ZISSERMAN's weak membrane model in computer vision [5]. This model can be translated into a simple nonlinear finite difference scheme (see CHAmbolle [13]) on a two-dimensional (in general, $d$-dimensional) grid. Its underlying discrete energy is of the form

$$
E_{\varepsilon}(u)=\frac{1}{2} \sum_{z_{1}, z_{2} \in \mathbb{Z}^{2} \cap \frac{1}{\varepsilon} Q,\left|z_{1}-z_{2}\right|=1} \min \left\{\left(u\left(z_{1}\right)-u\left(z_{2}\right)\right)^{2}, \varepsilon\right\}
$$


where $\varepsilon$ is the mesh size and $u$ represents the vertical displacement in the case of a membrane (or the grey level of an output picture in the applications to computer vision). The energy density can be seen as a truncated quadratic potential. In terms of the finite difference $u\left(z_{1}\right)-u\left(z_{2}\right) / \varepsilon$ it can be rewritten as

$$
\min \left\{\left(u\left(z_{1}\right)-u\left(z_{2}\right)\right)^{2}, \varepsilon\right\}=\varepsilon^{2} \min \left\{\left(\frac{u\left(z_{1}\right)-u\left(z_{2}\right)}{\varepsilon}\right)^{2}, \frac{1}{\varepsilon}\right\} .
$$

Its interpretation is that the "springs" of the discrete weak membrane behave linearly until the gradient (difference quotient) reaches the threshold $c_{\varepsilon}=1 / \sqrt{\varepsilon}$; after this threshold is reached, the spring is broken and the energy of the spring remains constant.

The overall behavior of such a system can be expressed by an asymptotic study of this energy as $\varepsilon \rightarrow 0$. Note that in the case of a (strong) discrete membrane the underlying energy is simply

$$
F_{\varepsilon}(u)=\frac{1}{2} \sum_{z_{1}, z_{2} \in \mathbb{Z}^{2} \cap \frac{1}{\varepsilon} Q,\left|z_{1}-z_{2}\right|=1}\left(u\left(z_{1}\right)-u\left(z_{2}\right)\right)^{2} .
$$

This is a convex energy and it is just a finite-difference approximation of the Dirichlet integral

$$
F(u)=\int_{Q}|\nabla u|^{2} \mathrm{~d} x .
$$

In the case of the weak membrane we are not in a convex setting; in particular, energies of the form $E_{\varepsilon}$ cannot be seen as finite approximations of integral energies defined on $H^{1}(Q)$. An asymptotic study of $E_{\varepsilon}$ has been performed by CHAMBOLLE [14] using the language of $\Gamma$-convergence (see $[7,16])$ and the techniques of Ambrosio and De Giorgi's SBV spaces (see [4, 17]). In loose terms, the space where the limit energy is finite consists of functions $u$ that are $H^{1}$ outside their set of discontinuities, which we denote by $S(u)$, and we have sufficient regularity to define a normal $v$ to $S(u)$. The $\Gamma$-limit of $E_{\varepsilon}$ can be written in this space as

$$
E(u)=\int_{Q}|\nabla u|^{2} \mathrm{~d} x+\int_{S(u)}|\nu|_{1} \mathrm{~d} \mathcal{H}^{1},
$$

where $|v|_{1}=\left|\left(v_{1}, v_{2}\right)\right|_{1}=\left|v_{1}\right|+\left|v_{2}\right|$ and $\mathcal{H}^{1}$ is the one-dimensional Hausdorff measure. The anisotropy in the "jump part" of the energy is clearly due to the anisotropy of the lattice structures. This energy highlights that the weak membrane may indeed undergo fracture in a fashion similar to Griffith brittle fracture [21], but the fracture energy is anisotropic since cracks follow the microscopical pattern of the lattice. Note that $E$ is an anisotropic version of the MumFord and SHAH functional of computer vision [26] to whom the weak membrane model is deeply connected.

In our setting we consider a random mixture of the two types of springs (the precise definition through the introduction of independent identically distributed random variables being found in Section 2), the strong springs with probability $p$ 
and the weak springs with probability $1-p$, the case $p=1$ corresponding to the discretization of the Dirichlet integral and the case $p=0$ corresponding to the weak membrane. We show the appearance of two regimes: if $p$ is larger than a percolation threshold $p_{c}$ then the effect of the weak springs is negligible; more precisely, we obtain that the $\Gamma$-limit is almost surely the Dirichlet integral. Conversely, if $p<p_{c}$ then the limit functional is defined on an SBV space, with a surface energy density depending on $p$. In this case, the limit energy takes the form

$$
E_{p}(u)=\int_{Q}|\nabla u|^{2} \mathrm{~d} x+\int_{S(u)} \lambda_{p}(v) \mathrm{d} \mathcal{H}^{1} .
$$

The result is deeply linked to percolation techniques. The proofs use arguments of $\Gamma$-convergence and geometric measure theory together with geometrical properties of percolation clusters. In the supercritical case we combine the compactness results in SBV and the rectifiability properties of the jump set $S(u)$ with the existence of many paths of strong connections to obtain that actually $S(u)$ is negligible. Conversely, in the subcritical case we use the properties of the "cluster of weak connections" to define the functions $\lambda_{p}$ and to construct test functions that provide the upper bound. The value $\lambda_{p}(v)$ is defined through the asymptotic behavior of the chemical distance (that is, the distance on the weak cluster) between a pair of points aligned with $v$. The general framework for first-passage percolation and chemical distance can be found in $[23,25]$. A crucial tool in the proof of the lower bound is the technical Lemma 1 that ensures that paths whose length is strictly less than the chemical distance contain a "substantial proportion" of strong connections. This result has been kindly provided to us by $\mathrm{H}$. Kesten, and its proof is contained in the Appendix.

It must be remarked that the corresponding problem in the case of deterministic homogenization, where we prescribe the microscopical periodic arrangement of the springs, can be performed by following the localization methods of $\Gamma$-convergence. In this case, the knowledge of averaged quantities such as the percentage of strong and weak springs only does not allow any accurate description of the limit energy; in particular, under proper choices of the local geometry, we can obtain both energies (1) and (2). In fact, we may consider the extreme periodic geometries of period $N$ where all weak connections are placed on the boundary of the periodicity cell. If the fraction of weak connections exceeds $1 / N$, then strong connections are completely isolated, and the arguments used by CHAMBOLLE [14] can be repeated to show that the limit is again the weak membrane energy. Conversely, if the positions of the weak and strong connections are reversed, then the strong connections constitute a connected frame that guarantees that the limit is defined on $H^{1}(Q)$, and hence, by a comparison argument it is the Dirichlet integral. Again note that we only need a fraction of $1 / N$ strong connections to construct such an example (in dimension $d$ actually only $N^{1-d}$ ). These considerations show that with fixed proportions of weak and strong connections different from 0 and 1 both strong and weak membrane models (and hence also the intermediate models) can be obtained; in particular an analysis giving optimal bounds for the limit energies such as that carried out by BRAIDES and FRANCFORT [10] for the homogenization of conducting networks cannot provide additional information. 
We note that there are many possible extensions of the weak membrane model to mechanically relevant system. On the one hand the limitation to antiplane displacements could be removed, so that the expected bulk term in the limit energy should then correspond in the subcritical case to a quadratic form of the strain and the function space would have to change from SBV to SBD (for the computation of the elastic constants for the bulk part in this context see the analog of the Chambolle result [14] by Alicandro et al. [3]). In that case the system may be interpreted as a thin discrete film (in the spirit of ALICANDRo et al. [1]), and additional anisotropies play a role in the determination of the surface part (see [3]). Furthermore, one may also consider frame-indifferent springs as in the model recently studied by FRIESECKE and THEIL [18]. In that framework an additional constraint of positive determinant for the displacement is added that should be then considered in the formulation of the limit surface energy. An intermediate case could be a "linearization" of the model in [18] as performed by BRAIDES et al. [12]. Note that all such models bring additional complex technical issues and have not been treated also in the deterministic case. Besides these many interesting variants of the problem, some fundamental issues remain to be explored even for our model, one of which is the asymptotical behavior of $\lambda_{p}$ as $p$ approaches increasingly the percolation threshold: whether $\lambda_{p} \rightarrow+\infty$ and whether $\lambda_{p}$ becomes "isotropic" close to the critical level.

\section{Notation and setup}

We will deal with limits of discrete models with randomly distributed nearest-neighbor interactions giving rise to free-discontinuity energies. In this section we recall the necessary background of percolation theory, $\Gamma$-convergence and the theory of special functions of bounded variation.

$\mathcal{L}^{d}$ denotes the $d$-dimensional Lebesgue measure and $\mathcal{H}^{k}$ the $k$-dimensional Hausdorff measure. We also use the notation $|A|=\mathcal{L}^{d}(A) . B_{\rho}(x)$ is the open ball of center $x$ and radius $\rho$.

\subsection{Special functions of bounded variation}

For the general theory of functions of bounded variation we refer to [4, 6]; here we just recall some definitions and results we shall use in the sequel. Let $Q$ be an open subset of $\mathbb{R}^{d}$. We say that $u \in L^{1}(Q)$ is a function of bounded variation if its distributional first derivatives $D_{i} u$ are (Radon) measures with finite total variation in $Q$. This space will be denoted by $B V(Q)$. We use $D u$ to indicate the vector-valued measure whose components are $D_{i} u$.

Let $u: Q \rightarrow \mathbb{R}$ be a Borel function. We say that $z \in \mathbb{R}$ is the approximate limit of $u$ in $x$ if for every $\varepsilon>0$

$$
\lim _{\rho \rightarrow 0^{+}} \rho^{-d}\left|\left\{y \in B_{\rho}(x) \cap Q:|u(y)-z|>\varepsilon\right\}\right|=0 .
$$

We define the jump set $S(u)$ offunction $u$ as the subset of $Q$ where the approximate limit of $u$ does not exist. It turns out that $S(u)$ is a Borel set and $|S(u)|=0$. If $u \in$ 
$B V(Q)$, then $S(u)$ is countably $(n-1)$-rectifiable; that is, $S(u)=N \cup\left(\bigcup_{i \in \mathbb{N}} K_{i}\right)$, where $\mathcal{H}^{d-1}(N)=0$ and $\left(K_{i}\right)$ is a sequence of compact sets, each contained in a $C^{1}$ hypersurface $\Gamma_{i}$. A normal unit vector $v_{u}$ to $S(u)$ exists $\mathcal{H}^{d-1}$-almost everywhere on $S(u)$, in the sense that, if $S(u)$ is represented as above, then $v_{u}(x)$ is normal to $\Gamma_{i}$ for $\mathcal{H}^{d-1}$-almost everywhere $x \in K_{i}$. Moreover, $v_{u}: S(u) \rightarrow S^{d-1}$ is a Borel function.

We say that a function $u \in B V(Q)$ is a special function of bounded variation if the singular part of $D u$ is concentrated on $S(u)$; that is, there exist $\phi \in\left(L^{1}(Q)\right)^{d}$ and $\psi \in\left(L^{1}\left(Q, \mathcal{H}^{d-1}\llcorner S(u))^{d}\right.\right.$ such that $D u=\phi \mathcal{L}^{d}+\psi \mathcal{H}^{d-1}\llcorner S(u)$. We denote the space of the special functions of bounded variation by $\operatorname{SBV}(Q)$. Note that if $\psi=0$ then $u \in W^{1,1}(Q)$. We denote by $\nabla u$ the density of the absolutely continuous part of $D u$ with respect to the Lebesgue measure (the function $\phi$ above). $\nabla u$ turns out to be the approximate differential of $u$, in the sense that

$$
\lim _{\rho \rightarrow 0^{+}} \rho^{-d} \int_{B_{\rho}(x) \cap Q} \frac{|u(y)-u(x)-\nabla u(x) \cdot(y-x)|}{|y-x|} \mathrm{d} y=0
$$

for almost every $x \in Q$.

A function $u: Q \rightarrow[-\infty,+\infty]$ is a generalized special function of bounded variation if its truncations are in $\operatorname{SBV}(Q)$; that is, for every $T>0$ we have $u_{T}:=$ $-T \wedge(u \vee T) \in \operatorname{SBV}(Q)$. This space is denoted by $\operatorname{GSBV}(Q)$. If $u \in \operatorname{GSBV}(Q)$ and $|\{|u|=+\infty\}|=0$ then $\nabla u=\lim _{T \rightarrow+\infty} \nabla u_{T}$ is defined almost everywhere and we set $S(u)=\bigcup_{T>0} S\left(u_{T}\right)$. Energies of the form

$$
\int_{Q} f(\nabla u) \mathrm{d} x+\int_{S(u)} g\left(v_{u}\right) \mathrm{d} \mathcal{H}^{d-1}
$$

with $f, g$ non-negative Borel functions are then well defined on such functions.

\section{2. $\Gamma$-convergence}

We recall the definition of $\Gamma$-convergence of a sequence of functionals $F_{j}$ defined on $\operatorname{GSBV}(Q)$ : we say that $\left(F_{j}\right) \Gamma$-converges to $F$ (on $\operatorname{GSBV}(Q)$ with respect to the convergence in measure) if for all $u \in \operatorname{GSBV}(Q)$

(i) (lower bound) for all sequences $\left(u_{j}\right)$ converging to $u$ in measure we have

$$
F(u) \leqq \liminf _{j} F_{j}\left(u_{j}\right)
$$

(ii) (upper bound) there exists a sequence $\left(u_{j}\right)$ converging to $u$ in measure such that

$$
F(u) \geqq \lim \sup _{j} F_{j}\left(u_{j}\right) .
$$

If (i) and (ii) hold then we write $F(u)=\Gamma-\lim _{j} F_{j}(u)$. We define the $\Gamma$-lower limit as

$$
\Gamma-\liminf { }_{j} F_{j}(u)=\inf \left\{\liminf _{j} F_{j}\left(u_{j}\right): u_{j} \rightarrow u\right\}
$$


and the $\Gamma$-upper limit as

$$
\Gamma-\lim \sup _{j} F_{j}(u)=\inf \left\{\limsup _{j} F_{j}\left(u_{j}\right): u_{j} \rightarrow u\right\},
$$

respectively. Then (i) also reads $F(u) \leqq \Gamma$ - $\liminf _{j} F_{j}(u)$ and (ii) reads $F(u) \geqq$ $\Gamma-\lim \sup _{j} F_{j}(u)$.

We will say that a family $\left(F_{\varepsilon}\right) \Gamma$-converges to $F$ if for all sequences $\left(\varepsilon_{j}\right)$ of positive numbers converging to 0 the conditions (i) and (ii) above are satisfied with $F_{\varepsilon_{j}}$ in place of $F_{j}$. The notation is modified accordingly. For an introduction to $\Gamma$-convergence we refer to $[7,16]$.

\subsection{Discrete-to-continuous limits}

The application of $\Gamma$-convergence to describe continuum limits of discrete systems has been used in different frameworks in various degrees of generality (see, for example, $[7,13,14,9,11,2])$. Here we will deal with a simple situation of nearest-neighbor energies in $\mathbb{R}^{2}$.

With fixed $\varepsilon>0$ we consider energies defined on functions parameterized on the lattice $\varepsilon \mathbb{Z}^{2} \cap Q$, or equivalently, upon scaling, on $\mathbb{Z}^{2} \cap \frac{1}{\varepsilon} Q$, of the form

$$
E_{\varepsilon}(u)=\frac{1}{2} \sum_{z_{1}, z_{2} \in \mathbb{Z}^{2} \cap \frac{1}{\varepsilon} Q,\left|z_{1}-z_{2}\right|=1} \varepsilon^{2} f_{\varepsilon}\left(z_{1}, z_{2}, \frac{u\left(\varepsilon z_{1}\right)-u\left(\varepsilon z_{2}\right)}{\varepsilon}\right) .
$$

with $u: \varepsilon \mathbb{Z}^{2} \cap Q \rightarrow \mathbb{R}$. We identify each such $u: \varepsilon \mathbb{Z}^{2} \rightarrow \mathbb{R}$ (extended to 0 outside $\left.\varepsilon \mathbb{Z}^{2} \cap Q\right)$ with the piecewise-constant extension $u(x)=u(\varepsilon z)$ if $x \in \varepsilon z+\left[-\frac{\varepsilon}{2}, \frac{\varepsilon}{2}\right)^{2}$, $z \in \mathbb{Z}^{2}$.

We will say that $E_{\varepsilon} \Gamma$-converge to $F$ if they $\Gamma$-converge as functionals defined on $\operatorname{GSBV}(Q)$ with the identification above (we set $E_{\varepsilon}(u)=+\infty$ if $u$ is not a piecewise-constant function as above).

Example 1. (1) (Quadratic potentials) In the trivial quadratic case $f_{\varepsilon}(i, j, z)=z^{2}$ then $E_{\varepsilon} \Gamma$-converge to the Dirichlet integral

$$
F(u)=\int_{Q}|\nabla u|^{2} \mathrm{~d} x
$$

on $H^{1}(Q)$ and $F(u)=+\infty$ if $u \in \operatorname{GSBV}(Q) \backslash H^{1}(Q)$.

(2) (Truncated quadratic potentials: see $[13,14,11])$ If $f_{\varepsilon}(i, j, z)=\min \left\{z^{2}, \frac{1}{\varepsilon}\right\}$ then $E_{\varepsilon} \Gamma$-converge to the weak membrane energy

$$
F(u)=\int_{Q}|\nabla u|^{2} \mathrm{~d} x+\int_{S(u)}|v|_{1} \mathrm{~d} \mathcal{H}^{1},
$$

where $|v|_{1}=\left|v_{1}\right|+\left|v_{2}\right|$.

(3) In both cases the sequence is equi-coercive, in the sense that from a sequence of functions bounded in measure with $\sup _{\varepsilon} E_{\varepsilon}\left(u_{\varepsilon}\right)<+\infty$ we may extract a subsequence converging to $u \in \operatorname{GSBV}(Q)$. 


\subsection{Percolation models}

The object of this paper is the asymptotic description of some models randomly mixing the two energy functionals in Example 1. Among the many ways to set up this problem in a stochastic framework, we will focus on the two percolation models described below. In both cases, a random choice is made for whether a bond (that is, the link between two neighboring points in the lattice) is "strong" (that is, the energy density between the two points is a quadratic potential) or "weak" (that is, it is a truncated quadratic potential as above).

2.4.1. Bond percolation model The first type of percolation model considered in this work consists in simply assigning the label "weak" or "strong" to a bond with probability $p$ and $1-p$, respectively, the choice being independent on distinct bonds. More precisely, this model is introduced as follows. Denote by $\hat{\mathbb{Z}}^{2}$ the set of middle points of the segments $\left[z_{1}, z_{2}\right], z_{1}, z_{2} \in \mathbb{Z}^{2},\left|z_{1}-z_{2}\right|=1$, of the standard integer grid $\mathbb{Z}^{2}$. Notice that $\hat{\mathbb{Z}}^{2}$ forms the dual grid of $\mathbb{Z}^{2}$ :

$$
\hat{\mathbb{Z}}^{2}=\left(\begin{array}{c}
\frac{1}{2} \\
\frac{1}{2}
\end{array}\right)+\left(\begin{array}{cc}
\frac{1}{2} & -\frac{1}{2} \\
\frac{1}{2} & \frac{1}{2}
\end{array}\right) \mathbb{Z}^{2} .
$$

The notation $z_{1}(\hat{z}), z_{2}(\hat{z})$ is used for the endpoints of the segment containing $\hat{z}$. We may identify each point in $\hat{z} \in \hat{\mathbb{Z}}^{2}$ with the corresponding closed segment $\left[z_{1}(\hat{z}), z_{2}(\hat{z})\right]$, so that points in $\hat{\mathbb{Z}}^{2}$ are identified with bonds in $\mathbb{Z}^{2}$.

Let $(\Omega, \mathcal{F}, \mathbf{P})$ be a probability space, and let $\left\{\xi_{\hat{z}}, \hat{z} \in \hat{\mathbb{Z}}^{2}\right\}$ be a family of independent identically distributed random variables such that

$$
\xi_{\hat{z}}= \begin{cases}\text { "weak" } & \text { with probability } 1-p, \\ \text { "strong" } & \text { with probability } p .\end{cases}
$$

In this way we associate to each bond of $\mathbb{Z}^{2}$ one of the labels "weak" or "strong". For brevity in what follows we identify "weak" and "strong" with the values 0 and 1 , respectively.

Given a bounded Lipschitz domain $Q \subset \mathbb{R}^{2}$, we will investigate the asymptotic behavior as $\varepsilon \rightarrow 0^{+}$of the functionals

$$
F_{\varepsilon}^{b, \omega}(u)=\sum_{\hat{z} \in \frac{1}{\varepsilon} Q \cap \hat{\mathbb{Z}}^{2}} \varepsilon \phi_{\hat{z}}^{\omega}\left(\frac{\left(u\left(\varepsilon z_{1}(\hat{z})\right)-u\left(\varepsilon z_{2}(\hat{z})\right)\right)^{2}}{\varepsilon}\right),
$$

where $u$ is a function defined on $\varepsilon \mathbb{Z}^{2} \cap Q$, and

$$
\phi_{\hat{z}}^{\omega}(s)= \begin{cases}s, & \text { if } \xi_{\hat{z}}(\omega)=1, \\ \min (s, 1), & \text { otherwise. }\end{cases}
$$

We will study the possible $\Gamma$-limits of $F_{\varepsilon}^{b, \omega}$, as $\varepsilon \rightarrow 0^{+}$, for different values of $p$ in (5). Note that this functional is of the form (3) with $f_{\varepsilon}\left(z_{1}, z_{2}, w\right)=\frac{1}{\varepsilon}$ $\phi_{\frac{z_{1}+z_{2}}{2}}^{\omega}\left(\varepsilon w^{2}\right)$. 
2.4.2. Site percolation model Another possible way of assigning a label "weak" or "strong" to bonds, is by instead randomly labeling the points of the lattice, and then assigning the label "strong" to a bond only if both its endpoints are labeled as "strong".

A standard site percolation model in $\mathbb{Z}^{2}$ is formed by a collection of independent identically distributed random variables $\xi_{z}, z \in \mathbb{Z}^{2}$, such that

$$
\xi_{z}= \begin{cases}0 \text { ("weak") } & \text { with probability } 1-p, \\ 1 \text { ("strong") } & \text { with probability } p .\end{cases}
$$

Then we define a random function

$$
\phi_{z_{1}, z_{2}}^{\omega}(s)= \begin{cases}0 & \text { if }\left|z_{1}-z_{2}\right| \neq 1 \\ s & \text { if }\left|z_{1}-z_{2}\right|=1 \text { and } \xi_{z_{1}}(\omega)=\xi_{z_{2}}(\omega)=1, \\ \min (s, 1) & \text { otherwise. }\end{cases}
$$

Given a bounded Lipschitz domain $Q \subset \mathbb{R}^{2}$, we introduce the functional

$$
F_{\varepsilon}^{s, \omega}(u)=\sum_{z_{1}, z_{2} \in \frac{1}{\varepsilon} Q \cap \mathbb{Z}^{2}} \varepsilon \phi_{z_{1}, z_{2}}^{\omega}\left(\frac{\left(u\left(\varepsilon z_{1}\right)-u\left(\varepsilon z_{2}\right)\right)^{2}}{\varepsilon}\right),
$$

where $u$ is a function defined on $\varepsilon \mathbb{Z}^{2} \cap Q$, and $\varepsilon$ is a small positive parameter. We will study the possible $\Gamma$-limits of $F_{\varepsilon}^{s, \omega}$, as $\varepsilon \rightarrow 0^{+}$, for different values of $p$.

Note that the functional $F_{\varepsilon}^{s, \omega}$ is of the form (3) with $f_{\varepsilon}\left(z_{1}, z_{2}, w\right)=\frac{1}{\varepsilon} \phi_{z_{1}, z_{2}}^{\omega}\left(\varepsilon w^{2}\right)$. Note also that in the notation of (3) the points $z_{i}$ with $\left|z_{1}-z_{2}\right| \neq 1$ are not considered, so that we may regard the function $\phi_{z_{1}, z_{2}}$ as having only two possible forms.

\subsection{Some results from percolation theory}

In this section we recall a number of percolation theory results which are formulated in the form adapted for our needs. We refer to the books by KESTEN [24] and GRIMMETT [22] for their proof if not stated otherwise.

We first consider the bond percolation model. We introduce a terminology for strong bonds; that is, those points $\hat{z} \in \hat{\mathbb{Z}}^{2}$ such that $\xi_{\hat{z}}=1$. Keeping in mind the identification of $\hat{z}$ with $\left[z_{1}(\hat{z}), z_{2}(\hat{z})\right]$ we say that two strong points $\hat{z}$ and $\hat{z}^{\prime}$ are adjacent if the corresponding two segments have an endpoint in common. A sequence of strong bonds $\hat{z}_{1}, \ldots, \hat{z}_{k}$ is said to be a strong path if any two consecutive points of this sequence are adjacent. In what follows we identify a strong path with the subset of $\mathbb{R}^{2}$ composed of the union of the corresponding segments. A subset $A$ of $\hat{\mathbb{Z}}^{2}$ of strong points is said to be connected if for every two points $\hat{z}^{\prime}, \hat{z}^{\prime \prime}$ of $A$ there exists a strong path as above such that $\hat{z}_{j} \in A, \hat{z}_{1}=\hat{z}^{\prime}, \hat{z}_{k}=\hat{z}^{\prime \prime}$. A maximum connected component of adjacent strong points is called a strong cluster.

Theorem 1. (Percolation threshold) For any $p<p_{c}:=1 / 2$ all the strong clusters are almost surely finite, while for any $p>1 / 2$ with probability one there is exactly one infinite strong cluster. 
The notation $S^{\delta}$ stands for a square of size $\delta$, centered at the origin, whose sides are not necessary parallel to the coordinate axis. A path joining two opposite sides of a square (or, more generally, of a bar) will be called a channel. Our analysis relies essentially on the following statement.

Theorem 2. (Channel property) Assume that $p>1 / 2$ (supercritical mode). Then there exist constants $c(p)>0$ and $c_{1}(p)>0$ such that almost surely for any $\delta$, $0<\delta \leqq 1$ there is a large enough number $N_{0}=N_{0}(\omega, \delta)$ such that for all $N>N_{0}$ and for any $S^{\delta}$ and $x_{0} \in[0,1]^{2}$ the square $N\left(S^{\delta}+x_{0}\right)$ contains at least $c(p) \delta N$ disjoint strong channels which connect opposite sides of the square. Moreover, the length of each such a channel does not exceed $c_{1}(p) \delta N$.

In the subcritical regime we need to introduce some terminology also for weak bonds; that is, those points $\hat{z} \in \hat{\mathbb{Z}}^{2}$ such that $\xi_{\hat{z}}=0$. In that case, we consider the shifted lattice $Z_{b}=\mathbb{Z}^{2}+\left(\frac{1}{2}, \frac{1}{2}\right)$ and notice that the set of middle points of its bonds coincides with $\hat{\mathbb{Z}}^{2}$ defined in (4). Thus, to each points $\hat{z} \in \hat{\mathbb{Z}}^{2}$ we can associate the corresponding bond in $Z_{b}$. If $\hat{z}$ is identified with the corresponding segment with endpoints in $Z_{b}$, then we may define the notion of adjacent points as for strong bonds. The notion of a weak channel and a weak cluster is modified accordingly. In what follows the notation $\mathcal{C}_{b}$ stands for the infinite weak cluster.

For $p<1 / 2$ there is almost surely a unique infinite weak cluster and the channel property stated above holds for the weak channels as well. Moreover, if we denote by $T_{v}^{\rho}$ the bar

$$
T_{v}^{\rho}=\left\{x \in \mathbb{R}^{2}:|\langle x, v\rangle| \leqq \rho, 0 \leqq\left\langle x, v^{\perp}\right\rangle \leqq 1\right\},
$$

where $0<\rho<1, v=\left(v_{1}, v_{2}\right) \in R^{2}$ is a unit vector and $v^{\perp}=\left(-v_{2}, v_{1}\right)$, then almost surely for sufficiently large $N$, in the $\operatorname{bar} N T_{v}^{\rho}$ there is a weak channel that links the smaller sides of the bar. Denote by $L_{N}=L_{N}(v, \rho, p, \omega)$ the length of the shortest such channel $\left(L_{N}=+\infty\right.$ if there is no such a channel), and

$$
\lambda^{b}(\nu, p)=\underset{\omega \in \Omega}{\operatorname{esssup}} \limsup \limsup _{\rho \rightarrow 0^{+}} \frac{L_{N}(v, \rho, p, \omega)}{N} .
$$

Denote by $\hat{y}_{0}$ and $\hat{y}_{N}(v)$ the elements of the infinite weak cluster $\mathcal{C}_{b}(\omega)$ such that

$$
\operatorname{dist}\left(0, \hat{y}_{0}\right)=\operatorname{dist}\left(0, \mathcal{C}_{b}\right), \quad \operatorname{dist}\left(N v, \hat{y}_{N}(v)\right)=\operatorname{dist}\left(N v, \mathcal{C}_{b}\right),
$$

and let $\tilde{L}_{N}(\nu, p, \omega)$ be the length of the shortest weak path which links $\hat{y}_{0}$ and $\hat{y}_{N}(v)$. According to $[19,20,27]$ the limits

$$
\lim _{N \rightarrow \infty} \frac{\tilde{L}_{N}(\nu, p, \omega)}{N} \text { and } \lim _{\rho \rightarrow 0^{+}} \lim _{N \rightarrow \infty} \frac{L_{N}(\nu, \rho, p, \omega)}{N}
$$

exist almost surely, and the following relation holds

$$
\lim _{N \rightarrow \infty} \frac{\tilde{L}_{N}(v, p, \omega)}{N}=\lim _{\rho \rightarrow 0^{+}} \lim _{N \rightarrow \infty} \frac{L_{N}(v, \rho, p, \omega)}{N}=\lambda^{b}(v, p) .
$$


Moreover, for $p<1 / 2$ we have $\lambda^{b}(v, p) \leqq c_{2}(p)$ with some constant $c_{2}(p)$. Conversely, it is easily seen that $\lambda^{b}(v, p) \geqq \bar{\lambda}^{b}(v, 1)=|v|_{1}$ for all $p$.

The following lemma (see the Appendix for a proof) expresses the fact that paths joining opposite sides of a bar with length less than the minimal one corresponding to that in the definition of $\lambda^{b}$, contain a substantial percentage of strong bonds.

Lemma 1. Let $\eta>0$ be fixed. Then there are $\rho \in(0,1)$ and $\delta>0$ such that almost surely there exists $N_{0}$ such that for all $N \geqq N_{0}$ and all channels $\left\{\hat{z}_{i}\right\}$ of length $L$ connecting the two shorter sides of $N T_{v}^{\rho}$ and with $L<\left(\lambda^{b}(v, p)-\eta\right) N$ we have $\#\left(\left\{i: \xi_{\hat{z}_{i}}=1\right\}\right) \geqq \delta(\eta) N$.

We now consider site percolation. We introduce a terminology for strong points; that is, those points $z \in \mathbb{Z}^{2}$ with $\xi_{z}=1$. Two strong points $z_{1}, z_{2} \in \mathbb{Z}^{2}$ are called adjacent if $\left|z_{1}-z_{2}\right|=1$. In this framework the corresponding notions of path, connectedness, and cluster are defined as for bond percolation. The analogue of Theorem 1 reads as follows.

Theorem 3. (Percolation threshold) There is a critical value $p_{c} \approx 0.59$ such that for any $p<p_{c}$ all the strong clusters are almost surely finite, while for any $p>p_{c}$ with probability one there is exactly one infinite strong cluster.

The statement of Theorem 2 remains valid as well, upon replacing the value $1 / 2$ with the site percolation threshold $p_{c}$.

In the subcritical regime we need to introduce some terminology also for weak points; that is, those $z \in \mathbb{Z}^{2}$ with $\xi_{z}=0$. The situation is not symmetric to that of strong points, since in order to have a weak interaction between two points we only need that at least one of the two is a weak point. The notion of adjacent weak point is then modified; namely, we say that two weak vertices $z_{1}$ and $z_{2}$ are adjacent if $0<\left|z_{1}-z_{2}\right| \leqq \sqrt{2}$. The notion of a weak channel and a weak cluster is modified accordingly. Again, for $p<p_{c}$ there is almost surely a unique unbounded weak cluster and the channel property holds for weak channels. We may then define $\lambda^{s}$ analogously to $\lambda^{b}$ in (7). The statement of Lemma 1 also holds true for the site percolation model.

Note that, in contrast with bond percolation, in the case of the site percolation model the presence of a weak channel in some direction creates a topological obstacle for the existence of a strong transversal channel.

\section{Main results}

\subsection{Bond percolation model}

The structure of the $\Gamma$-limit functional depends crucially on whether $p<1 / 2$ or $p>1 / 2$ (recall that in dimension two the percolation threshold $p_{c}$ is $1 / 2$ ). We have the following result. 
Theorem 4. (i) (supercritical regime) Let $p>1 / 2$. Then almost surely

$$
\Gamma-\lim _{\varepsilon \rightarrow 0} F_{\varepsilon}^{b, \omega}(u)= \begin{cases}\int_{Q}|\nabla u|^{2} \mathrm{~d} x, & \text { if } u \in H^{1}(Q), \\ +\infty, & \text { otherwise }\end{cases}
$$

on the space of GSBV functions.

(ii) (subcritical regime) If $p<1 / 2$ then almost surely we have

$$
\Gamma-\limsup _{\varepsilon \rightarrow 0^{+}} F_{\varepsilon}^{b, \omega}(u)=\int_{Q}|\nabla u|^{2} \mathrm{~d} x+\int_{S(u)} \lambda^{b}(v(x), p) \mathrm{d} \mathcal{H}^{1}
$$

for all $u \in \operatorname{GSBV}(Q)$, where $\lambda^{b}(v, p)$ has been defined in (7).

Before proving the results above we note the following coerciveness property, which in particular implies that the domain of the $\Gamma$-limit is always contained in $\operatorname{GSBV}(Q)$.

Proposition 1. (Equi-coerciveness and lower bound) Let $p \in[0,1]$ and $\omega \in \Omega$; then

$$
\Gamma-\liminf _{\varepsilon \rightarrow 0} F_{\varepsilon}^{b, \omega}(u) \geqq \int_{Q}|\nabla u|^{2} \mathrm{~d} x+\int_{S(u)}|\nu|_{1} \mathrm{~d} \mathcal{H}^{1},
$$

where $|v|_{1}=\left|v_{1}\right|+\left|v_{2}\right|$. Moreover if $\left(\varepsilon_{j}\right)$ converges to 0 , then for all $\left(u_{j}\right)$ bounded in measure such that $\sup _{j} F_{\varepsilon_{j}}^{b, \omega}\left(u_{j}\right)<+\infty$ there exists a subsequence of $\left(u_{j}\right)$ converging in measure to some $u \in \operatorname{GSBV}(Q)$.

Proof. It suffices to remark that for all $p \in[0,1], \omega \in \Omega$, and $u$ we have $F_{\varepsilon}^{b, \omega}(u) \geqq$ $E_{\varepsilon}(u)$, where $E_{\varepsilon}$ is as in Example 1(2). It suffices then to use the results recalled in items (2) and (3) in Example 1.

Remark 1. Note that for all $p \in[0,1], \omega \in \Omega$, and $u$ we have $F_{\varepsilon}^{b, \omega}(u) \leqq E_{\varepsilon}(u)$, where $E_{\varepsilon}$ is as in Example 1(1). By taking the $\Gamma$-lim sup in this inequality, if $u \in H^{1}(Q)$ we get $\Gamma$-lim $\sup _{\varepsilon \rightarrow 0} F_{\varepsilon}^{b, \omega}(u) \leqq \int_{Q}|\nabla u|^{2} \mathrm{~d} x$. By (9) it then follows that the $\Gamma$-limit always exists in $H^{1}(Q)$ and

$$
\Gamma-\lim _{\varepsilon \rightarrow 0} F_{\varepsilon}^{b, \omega}(u)=\int_{Q}|\nabla u|^{2} \mathrm{~d} x \quad \text { for all } u \in H^{1}(Q) .
$$

Proof. [of Theorem 4(i)] We say that an element $\omega \in \Omega$ (or, equivalently a "realization" of the medium) is typical if the statement of Theorem 2 holds. Fix a typical realization $\omega$, and let $u^{\varepsilon} \rightarrow u$ be a sequence converging in measure and with bounded energy; that is, $F_{\varepsilon}^{b, \omega}\left(u^{\varepsilon}\right) \leqq C$. Recall that we identify discrete functions $u^{\varepsilon}$ with the corresponding piecewise-constant functions of continuous argument: $u^{\varepsilon}(x)=u^{\varepsilon}(z(x))$ with $x \in \mathbb{R}^{2}$ and $z(x) \in \mathbb{Z}^{2}$ defined almost everywhere by $|z(x)-x|=\operatorname{dist}\left(x, \mathbb{Z}^{2}\right)$. For the extended function we keep the same notation $u^{\varepsilon}$. Note that $u \in \operatorname{GSBV}(Q)$ by Proposition 1 . By Remark 1 to prove the thesis of the theorem it suffices to show that $u \in H^{1}(Q)$. 
Suppose that $u \notin H^{1}(Q)$, and denote by $u_{t}^{\varepsilon}$ the truncation of $u^{\varepsilon}$ at the level $t$ :

$$
u_{t}^{\varepsilon}(x)=\min \left(t, \max \left(-t, u^{\varepsilon}\right)\right) .
$$

For any $t>0$ the sequence $u_{t}^{\varepsilon}$ is of bounded energy since $F_{\omega}^{b, \varepsilon}\left(u_{t}^{\varepsilon}\right) \leqq F_{\omega}^{b, \varepsilon}\left(u^{\varepsilon}\right)$, and $u_{t}^{\varepsilon} \rightarrow u_{t} \in \operatorname{SBV}(Q)$ in $L^{2}(Q)$. Under our assumptions, the function $u_{t}$ does not belong to $H^{1}(Q)$ for sufficiently large $t$; that is, the discontinuity set $S\left(u_{t}\right)$ is not empty and $\mathcal{H}^{1}\left(S\left(u_{t}\right)\right)>0$.

By the properties of $S\left(u_{t}\right)$ (see [4]) for $\mathcal{H}^{1}$-almost all $x_{0} \in S\left(u_{t}\right)$ there exist two numbers $u^{+}=u^{+}\left(x_{0}\right)$ and $u^{-}=u^{+}\left(x_{0}\right), u^{+} \neq u^{-}$, and $v=v_{u}\left(x_{0}\right)$ such that, in the unit square $S_{v}$ centered in 0 and with one side parallel to $v$, the following relation holds

$$
\lim _{\eta \rightarrow 0^{+}} \int_{S_{v}}\left|u_{t}\left(\frac{x-x_{0}}{\eta}\right)-u_{0}(x)\right| \mathrm{d} x=0
$$

where

$$
u_{0}(x)= \begin{cases}u^{+} & \text {if }\langle x, v\rangle>0 \\ u^{-} & \text {if }\langle x, v\rangle<0\end{cases}
$$

Since $u_{t}^{\varepsilon}$ converges in $L^{2}\left([0,1]^{2}\right)$ to $u_{t}$, as $\varepsilon \rightarrow 0^{+}$, this yields

$$
\lim _{\eta \rightarrow 0^{+}} \lim _{\varepsilon \rightarrow 0^{+}} \int_{S_{v}}\left|u_{t}^{\varepsilon}\left(\frac{x-x_{0}}{\eta}\right)-u_{0}(x)\right| \mathrm{d} x=0 .
$$

Denote $I^{ \pm}=\left\{x \in S_{v}:\langle x, v\rangle= \pm 1 / 2\right\}$, the two sides of $S_{v}$ orthogonal to $v$. Taking appropriate subsequences $\eta \rightarrow 0^{+}$and $\varepsilon \rightarrow 0^{+}$, one may assume without loss of generality that

$$
\lim _{\eta \rightarrow 0^{+}} \lim _{\varepsilon \rightarrow 0^{+}} \mathcal{H}^{1}\left\{x \in I^{ \pm}:\left|u_{t}^{\varepsilon}\left(\frac{x-x_{0}}{\eta}\right)-u^{ \pm}\right|>\frac{u^{+}-u^{-}}{8}\right\}=0 .
$$

We choose $\eta$ and then $\varepsilon_{0}$ in such a way that

$$
\mathcal{H}^{1}\left\{x \in I^{ \pm}:\left|u_{t}^{\varepsilon}\left(\frac{x-x_{0}}{\eta}\right)-u^{ \pm}(x)\right|>\frac{u^{+}-u^{-}}{8}\right\}<\frac{1}{8} c(p) .
$$

for $\varepsilon<\varepsilon_{0}$.

By Theorem 2 for small enough $\varepsilon$ in the square $\eta S_{v}+x_{0}$ there are at least $c(p) \eta / \varepsilon$ disjoint strong channels that connect the sides $I^{-}$and $I^{+}$. Denote these channels by $K_{1}, K_{2}, \ldots, K_{M}, M \geqq c(p) \eta / \varepsilon$, and the vertices of each $K_{j}$ by $z_{1}^{j}, z_{2}^{j}, \ldots, z_{l_{j}}^{j}$ with $l_{j} \leqq c_{1}(p) \eta / \varepsilon$ and $z_{i}^{j} \in \varepsilon \mathbb{Z}^{2}$.

Considering (11) and slightly reducing the constant $c(p)$ we may suppose that all the channels possess the following properties

$$
u_{t}^{\varepsilon}\left(z_{1}^{j}\right) \leqq u^{-}+\frac{u^{+}-u^{-}}{4}, \quad u_{t}^{\varepsilon}\left(z_{l_{j}}^{j}\right) \geqq u^{+}-\frac{u^{+}-u^{-}}{4} .
$$


Therefore, for each channel $K_{j}$ one has

$$
\begin{aligned}
\frac{\left(u^{+}-u^{-}\right)^{2}}{4} & \leqq\left(u_{t}^{\varepsilon}\left(z_{l_{j}}^{j}-u_{t}^{\varepsilon}\left(z_{1}^{j}\right)\right)^{2}=\left(\sum_{i=1}^{l_{j}-1}\left(u\left(z_{i+1}^{j}\right)-u\left(z_{i}^{j}\right)\right)\right)^{2}\right. \\
& \leqq l_{j} \sum_{i=1}^{l_{j}-1}\left(u_{t}^{\varepsilon}\left(z_{i+1}^{j}\right)-u_{t}^{\varepsilon}\left(z_{i}^{j}\right)\right)^{2} \\
& \leqq c_{1}(p) \frac{\eta}{\varepsilon} \sum_{i=1}^{l_{j}-1}\left(u_{t}^{\varepsilon}\left(z_{i+1}^{j}\right)-u_{t}^{\varepsilon}\left(z_{i}^{j}\right)\right)^{2}
\end{aligned}
$$

This implies the inequality

$$
\sum_{i=1}^{l_{j}-1}\left(u_{t}^{\varepsilon}\left(z_{i+1}^{j}\right)-u_{t}^{\varepsilon}\left(z_{i}^{j}\right)\right)^{2} \geqq \frac{\left(u^{+}-u^{-}\right)^{2}}{4} \frac{\varepsilon}{\eta} \frac{1}{c_{1}(p)} .
$$

Summing up over the channels gives

$$
\sum_{j=1}^{M} \sum_{i=1}^{l_{j}-1}\left(u_{t}^{\varepsilon}\left(z_{i+1}^{j}\right)-u_{t}^{\varepsilon}\left(z_{i}^{j}\right)\right)^{2} \geqq \frac{\left(u^{+}-u^{-}\right)^{2}}{4} \frac{\varepsilon}{\eta} \frac{c(p)}{c_{1}(p)} \frac{\eta}{\varepsilon}=\frac{\left(u^{+}-u^{-}\right)^{2}}{4} \frac{c(p)}{c_{1}(p)} .
$$

Clearly, since the channels are disjoint, the total energy of the square $S_{v}^{\eta}+x_{0}$ is estimated from below as follows

$$
\begin{aligned}
\sum_{z_{1}, z_{2} \in\left(S_{v}^{\eta}+x_{0}\right) \cap \varepsilon \mathbb{Z}^{2}} \varepsilon \phi_{\hat{z}}\left(\frac{\left(u^{\varepsilon}\left(z_{1}\right)-u^{\varepsilon}\left(z_{2}\right)\right)^{2}}{\varepsilon}\right) & \geqq \sum_{j=1}^{M} \sum_{i=1}^{l_{j}-1}\left(u_{t}^{\varepsilon}\left(z_{i+1}^{j}\right)-u_{t}^{\varepsilon}\left(z_{i}^{j}\right)\right)^{2} \\
& \geqq \frac{\left(u^{+}-u^{-}\right)^{2}}{4} \frac{1}{c_{1}(p)} c(p) .
\end{aligned}
$$

Since this estimate is $\eta$-independent, we may fix an arbitrary number $N$ of distinct points of $S\left(u_{t}\right)$ as above with $\left(u^{+}-u^{-}\right) \geqq C>0$, and repeat the arguments above on disjoint squares centered at these points. We then obtain the estimate for the total energy

$$
F_{\varepsilon}^{b, \omega}\left(u^{\varepsilon}\right) \geqq N \frac{C^{2}}{4} \frac{c(p)}{c_{1}(p)},
$$

which contradicts our assumption that the energy is bounded. Hence we must have $\mathcal{H}^{1}\left(S\left(u_{t}\right)\right)=0$ for all $t$, which implies that $u \in H^{1}(Q)$.

Remark 2. The statement (i) of Theorem 4 remains valid in any dimension $d \geqq 2$. Indeed, the assertions of Theorems 1 and 2 hold true for any $d \geqq 2$, and the proof of item (i) above can be easily rearranged so that it applies for any $d \geqq 2$. Notice that for $d>2$ we have $p_{c}<1 / 2$. 
Proof. [of Theorem 4(ii)] We first prove the liminf inequality. It suffices to deal with $u \in \operatorname{SBV}(Q)$ since by comparison the limit energy is $+\infty$ outside $\operatorname{GSBV}(Q)$ (Proposition 1) and it is not restrictive to suppose that $u \in L^{\infty}(Q)$ by a truncation argument.

Let $u_{\varepsilon} \rightarrow u$ be such that $F_{\varepsilon}^{b, \omega}\left(u_{\varepsilon}\right) \leqq c<+\infty$. We will give an estimate on the energy related to $u_{\varepsilon}$ concentrating at almost every point in $S(u)$. We fix $R>0$ and $\gamma>0$; we will show that

$$
\Gamma-\liminf _{\varepsilon \rightarrow 0^{+}} F^{b, \omega}\left(u_{\varepsilon}\right) \geqq \int_{S(u) \cap\left\{u^{+}-u^{-} \mid>1 / R\right\}}\left(\lambda^{b}\left(v_{u}(x), p\right)-\gamma\right) \mathrm{d} \mathcal{H}^{1} .
$$

From this estimate the complete liminf inequality is easily recovered.

For $\gamma$ as above let $\delta=\delta(\gamma / 2)>0$ be defined by Lemma 1. Consider $x \in S(u)$ and $\rho>0$. We denote by $T_{\rho, k}(x)$ the bar

$$
T_{\rho, k}(x)=\left\{y \in \mathbb{R}^{2}:\left|\left\langle(y-x), v_{u}(x)\right\rangle\right|<\frac{1}{2} k \rho,\left|\left\langle x, v^{\perp}\right\rangle\right|<\frac{1}{2} \rho\right\},
$$

and by

$$
\begin{aligned}
T_{\rho, k}^{ \pm}(x) & =\left\{y \in \mathbb{R}^{2}:\left|\left\langle(y-x), v_{u}(x)\right\rangle\right| \leqq \frac{1}{2} k \rho,\left\langle x, v^{\perp}\right\rangle= \pm \frac{1}{2} \rho\right\}, \\
T_{\rho, k}^{ \pm, \perp}(x) & =\left\{y \in \mathbb{R}^{2}:\left\langle(y-x), v_{u}(x)\right\rangle=\frac{1}{2} k \rho,\left|\left\langle x, v^{\perp}\right\rangle\right| \leqq \frac{1}{2} \rho\right\}
\end{aligned}
$$

its four sides. We choose $k=\delta / 16$.

We choose $x$ and $\rho$ such that

(1) $\left|u^{+}(x)-u^{-}(x)\right| \geqq \frac{1}{R}$;

(2) $\rho<\frac{\delta}{4} \frac{1}{R^{2}} \frac{1}{\lambda^{b}\left(v_{u}(x), p\right)}$;

(3) $\mathcal{H}^{1}\left(\left\{x \in T_{\rho, k}^{ \pm}(x):\left|u^{+}(x)-u^{-}(x)\right|>\frac{1}{8 R}\right\}\right) \leqq \frac{\delta}{8} \rho$;

(4) $u_{\varepsilon} \rightarrow u$ in $L^{1}\left(\partial T_{\rho, k}(x)\right)$.

In order to simplify the notation, it is not restrictive to suppose that $x=0$. We scale our problem by the factor $N=\frac{\rho}{\varepsilon}$ to the bar $T_{N, k}(0)$ and the corresponding $v_{\varepsilon}(x)=u_{\varepsilon}(\varepsilon x)$.

Let $I=I(\varepsilon)$ be the set of all $\hat{z} \in T_{N, k}(0)$ such that

$$
\text { (i) } \xi_{\hat{z}}=0 ; \quad \text { (ii) }\left|u_{\varepsilon}\left(\varepsilon z_{1}(\hat{z})\right)-u_{\varepsilon}\left(\varepsilon z_{2}(\hat{z})\right)\right| \geqq \sqrt{\varepsilon} \text {. }
$$

Suppose that

$$
\#(I(\varepsilon))<\left(\lambda^{b}\left(v_{u}(x), p\right)-\gamma\right) N .
$$

We then construct strong channels as follows. Let

$$
\begin{gathered}
J_{0}=\bigcup\left\{S \text { closed unit cube with integer vertices, } S \cap T_{N, k}^{-, \perp}(0) \neq \emptyset\right\}, \\
J_{i}^{\perp}=\bigcup_{\hat{z} \in J_{i}} \hat{z}^{\perp}
\end{gathered}
$$


(we use the general notation $A^{\perp}=\bigcup_{\hat{z} \in A} \hat{z}^{\perp}$ ),

$$
\begin{aligned}
\widetilde{J}_{i}^{\perp} & =J_{i}^{\perp} \cup\left\{\hat{z}: \exists \text { path in } I^{\perp} \text { connecting } \hat{z}^{\perp} \text { and } J_{i}^{\perp}\right\}, \\
J_{i+1} & =\bigcup\left\{S \text { closed unit cube with integer vertices, } S \cap \widetilde{J}_{i}^{\perp} \neq \emptyset\right\} .
\end{aligned}
$$

We finally define $K_{i}$ as the unique path in $\partial J_{i} \cap T_{N, k}(0)$ joining $T_{N, k}^{+}(0)$ and $T_{N, k}^{-}(0)$, and $M$ as the maximal index such that

$$
K_{M-1} \cap T_{N, k}^{+, \perp}(0)=\emptyset .
$$

By construction, $K_{1}, \ldots, K_{M}$ are disjoint strong channels connecting $T_{N, k}^{+}(0)$ and $T_{N, k}^{-}(0)$. Moreover, for each element in $\left(K_{i}\right)^{\perp}$ there exists a path with exactly $i$ elements in the complement of $I^{\perp}$ connecting it with $J_{0}$.

We conclude that there exists a path in $\hat{Z}^{\perp}$ connecting $T_{N, k}^{-, \perp}(0)$ and $T_{N, k}^{+, \perp}(0)$ with exactly $M$ elements from the complement of $I^{\perp}$. From Lemma 1 we then deduce that $M>\delta N$.

Note that in order to estimate the limit of $F_{\varepsilon}^{b, \omega}\left(u_{\varepsilon}, T_{\rho, k}(x)\right)$ we may set $\xi_{\hat{z}}^{\varepsilon}=1$ for all $\hat{z} \notin I(\varepsilon)$. We can then repeat the argument in the proof of theorem 4 and conclude that

$$
\liminf _{\varepsilon \rightarrow 0^{+}} F_{\varepsilon}^{b, \omega}\left(u_{\varepsilon}, T_{\rho, k}(x)\right) \geqq C,
$$

with $C=C(\gamma, R)$. Since this inequality may hold only for a finite number of $x$, we conclude that (13) must fail for almost all $x$, so that

$$
\#(I(\varepsilon)) \geqq\left(\lambda^{b}\left(v_{u}(x), p\right)-\gamma\right) N,
$$

from which we deduce that

$$
\liminf _{\varepsilon \rightarrow 0^{+}} F_{\varepsilon}^{b, \omega}\left(u_{\varepsilon}, T_{\rho, k}(x)\right) \geqq\left(\lambda^{b}\left(v_{u}(x), p\right)-\gamma\right) \rho,
$$

and inequality (12).

We now prove the limsup inequality. By the density results by CoRTESANI and ToAder [15] (see also [8]) the space $\operatorname{GSBV}(Q)$ can also be seen as the closure of piecewise-smooth functions in the sense that for all $u \in \operatorname{GSBV}(Q)$ with $\nabla u \in L^{2}(Q)$ and $\mathcal{H}^{1}(S(u))<+\infty$ there exists a sequence of finite families of disjoint closed segments $\left(P_{j}^{i}\right)$ and functions $u_{j}$ that are $C^{\infty}$ and Lipschitz outside $\bigcup_{i} P_{j}^{i}$ converging to $u$ in measure such that

$\underset{j}{\lim \sup }\left(\int_{Q} f\left(\nabla u_{j}\right) \mathrm{d} x+\int_{S\left(u_{j}\right)} g\left(v_{u_{j}}\right) \mathrm{d} \mathcal{H}^{1}\right) \leqq \int_{Q} f(\nabla u) \mathrm{d} x+\int_{S(u)} g\left(v_{u}\right) \mathrm{d} \mathcal{H}^{1}$

for all continuous $f, g$ with $|f(z)| \leqq C\left(1+|z|^{2}\right)$. This immediately follows from Theorem 3.1 and Remarks 3.2 and 3.5 in [15] and the continuity of $U \mapsto$ $\int_{Q} f(U) \mathrm{d} x$ in $L^{2}$. 
Using this observation, it suffices to prove the limsup inequality of Theorem 4(ii) only for piecewise-smooth functions as above. In fact, by the lower semicontinuity of the $\Gamma$-upper limit (see, for example, [7, Sect. 1.7]), from (14), applied to $f(w)=|w|^{2}$ and $g(w)=\lambda^{b}(w, p)$, we get

$$
\begin{aligned}
\Gamma-\limsup _{\varepsilon \rightarrow 0^{+}} F_{\varepsilon}^{b, \omega}(u) & \leqq \liminf _{j}\left(\Gamma-\limsup _{\varepsilon \rightarrow 0^{+}} F_{\varepsilon}^{b, \omega}\left(u_{j}\right)\right) \\
& \leqq \limsup _{j}\left(\int_{Q}\left|\nabla u_{j}\right|^{2} \mathrm{~d} x+\int_{S\left(u_{j}\right)} \lambda^{b}\left(v_{u_{j}}, p\right) \mathrm{d} \mathcal{H}^{1}\right) \\
& \leqq \int_{Q}|\nabla u|^{2} \mathrm{~d} x+\int_{S(u)} \lambda^{b}\left(v_{u}, p\right) \mathrm{d} \mathcal{H}^{1}
\end{aligned}
$$

as desired.

We then have to construct a recovery sequence for the $\Gamma$-limsup for a piecewise-smooth function. Since the construction is performed by modifying the target function $u$ close to each segment of $S(u)$, we may deal with $S(u)$ composed of only one segment. It is no restriction to consider only the case $S(u)=[-1,1] \times\{0\}$. Note that the traces of $u$ on both sides of $S(u)$ are well-defined Lipschitz functions. We can also suppose that

$$
\begin{array}{ll}
u\left(x^{1}, x^{2}\right)=u^{+}\left(x^{1}\right) & \text { on }[-1-\eta, 1+\eta] \times(0, \eta), \\
u\left(x^{1}, x^{2}\right)=u^{-}\left(x^{1}\right) & \text { on }[-1-\eta, 1+\eta] \times(-\eta, 0),
\end{array}
$$

for some $\eta>0$.

Fix arbitrary small $\delta>0, \delta<\eta$. Then by (8), almost surely for all sufficiently small $\varepsilon>0$ in the rectangle $R_{\delta}^{\eta}=[-1-\eta, 1+\eta] \times[-\delta, \delta]$ there is a non selfintersecting weak channel $K_{\varepsilon} \subset \varepsilon \mathbb{Z}_{b}$ connecting the left and the right sides of the rectangle, of length no greater than $2 \lambda^{b}\left(e_{1}, p\right)(1+\eta)+O(\delta)+o(1)$, where $o(1)$ tends to zero as $\varepsilon \rightarrow 0$.

We define

$$
\tilde{u}^{\varepsilon}= \begin{cases}u(x) & \text { if } x \notin R_{\delta}^{\eta}, \\ u^{+}(x) & \text { if } x \text { belongs to the closure of the connected component of } \\ & R_{\delta}^{\eta} \text { containing the upper side } \\ u^{-}(x) & \text { otherwise. }\end{cases}
$$

By construction, the jump set of $\tilde{u}^{\varepsilon}$ in $R_{\delta}^{\eta}$ coincides with $K_{\varepsilon}$; that is, $S\left(\tilde{u}^{\varepsilon}\right) \cap R_{\delta}^{\eta}$ consists of all weak bonds in $\varepsilon \mathbb{Z}_{b}$ which belong to $K_{\varepsilon}$. Clearly, $\mathcal{H}^{1}\left(S\left(\tilde{u}^{\varepsilon}\right) \cap R_{\delta}^{\eta}\right)=$ $2 \lambda^{b}\left(e_{1}, p\right)(1+\eta)+o(1)$.

Let $u^{\varepsilon}$ be the discretization of $\tilde{u}^{\varepsilon}$. Then the function $u^{\varepsilon}$ has a "jump" along the channel $K_{\varepsilon}$, and after a straightforward computation taking into account the fact that in the complement to $K_{\varepsilon}$ the function $u^{\varepsilon}$ is Lipschitz continuous, we have 


$$
\begin{aligned}
F_{\varepsilon}^{b, \omega}\left(u^{\varepsilon}\right) & \leqq \int_{Q \backslash K_{\varepsilon}}|\nabla u|^{2} \mathrm{~d} x+\left|K_{\varepsilon}\right| \\
& =\int_{Q}|\nabla u|^{2} \mathrm{~d} x+2 \lambda^{b}\left(e_{2}, p\right)(1+\eta)+O(\delta)+o(1) \\
& =\int_{Q}|\nabla u|^{2} \mathrm{~d} x+\lambda^{b}\left(e_{2}, p\right) \mathcal{H}^{1}(s(u))(1+\eta)+O(\delta)+o(1),
\end{aligned}
$$

with $o(1) \rightarrow 0$ as $\varepsilon \rightarrow 0$. Since $\eta$ and $\delta$ are arbitrary positive numbers, this implies the desired bound.

\subsection{Site percolation model}

The results below are basically the same as those obtained for the bond percolation model. We only formulate these results and leave their proof to the reader.

In the supercritical regime $p>p_{c}$ the structure of the $\Gamma$-limit of $F_{\varepsilon}^{s, \omega}$ is described by the following statement:

Theorem 5. (i) (supercritical regime) Let $p>p_{c}$. Then almost surely

$$
\Gamma-\lim _{\varepsilon \rightarrow 0} F_{\varepsilon}^{s, \omega}(u)= \begin{cases}\int_{Q}|\nabla u|^{2} \mathrm{~d} x, & \text { if } u \in H^{1}(Q), \\ +\infty, & \text { otherwise. }\end{cases}
$$

The limit functional is defined on $G S B V(Q)$.

(ii) (subcritical regime) If $p<p_{c}$ then almost surely we have

$$
\Gamma-\lim _{\varepsilon \rightarrow 0^{+}} F_{\varepsilon}^{s, \omega}(u)=\int_{Q}|\nabla u|^{2} \mathrm{~d} x+\int_{S(u)} \lambda^{s}(v(x), p) \mathrm{d} \mathcal{H}^{1}
$$

on $\operatorname{GSBV}(Q)$, where $\lambda^{s}(\nu, p)$ has been defined as in (7).

\section{Appendix}

This appendix contains a proof of Lemma 1 kindly provided by H. Kesten. It relies on the following large deviation result for Bernoulli percolation at $p>p_{c}$ (see GaRET and MARCHAND [20]). We denote by $D(x, y)$ the chemical distance between $x$ and $y$ for $x, y \in \mathbb{Z}^{d}$, that is, the length of the shortest weak path which connects $x$ and $y$, and by $\|\cdot\|$ the norm $\|x\|=\sum_{i=1}^{d}\left|x_{i}\right|$. Differently from the previous sections we will use the terminology "black" and "white" bonds in place of "strong" and "weak" bonds, respectively.

Proposition 2. ([20]) There exists a norm $\mu(x)$ such that for all $\varepsilon>0$

$$
\limsup _{\|x\| \rightarrow \infty} \frac{\log P\left\{\mathbf{0} \leftrightarrow x, \frac{D(\mathbf{0}, x)}{\mu(x)} \notin(1-\varepsilon, 1+\varepsilon)\right\}}{\|x\|}<0,
$$

where $y \leftrightarrow x$ stands for the event that both $x$ and $y$ are elements of the infinite cluster. 
We need a number of definitions. Let

$$
\begin{aligned}
& \mathcal{E}(v, N, \delta, \eta)=\{\text { there exists a path from } \mathbf{0} \text { to } N v \text { of length } \leqq N(\alpha-\delta) \\
& \text { and containing at most } \eta N \text { black edges }\} \text {. }
\end{aligned}
$$

Here $v \in \mathbb{R}^{d} \backslash \mathbf{0}, \alpha=\mu(v)$. Further, let $M$ be some large integer and $\mathbf{i}=$ $\left(i_{1}, i_{2}, \ldots, i_{d}\right) \in \mathbb{Z}^{d}$, and define

$$
B(\mathbf{i})=B_{M}(\mathbf{i})=\prod_{k=1}^{d}\left[i_{k}-M, i_{k}+M\right]
$$

(a closed cube of size $2 M$ with center at $\mathbf{i})$. Let $\mathbf{w}=w(0)=\mathbf{0}, w(1), \ldots, w(r)$ be a sequence of vertices in $\mathbb{Z}^{d}$ for which

$$
w(j+1) \in \partial B(w(j)), \text { or equivalently }\|w(j+1)-w(j)\|=M, 0 \leqq j \leqq r-1
$$

( $\partial B$ denotes the topological boundary of $B$ ), and

$$
[N v]:=(\text { nearest lattice point to } N v) \in B(w(r)) .
$$

In addition, let $\mathcal{S}$ be a subsequence of $0,1, \ldots, r$, say $\mathcal{S}=\left\{j_{1}, \ldots, j_{t}\right\}$ with $\left.0 \leqq j_{1}<j_{2}<\cdots<j_{t} \leqq r\right\}$ and define the event

$\mathcal{F}(\mathbf{w}, \mathcal{S})=\left\{\right.$ there exists a path $\sigma$ on $\mathbb{Z}^{d}$ with properties (17)-(21) below $\}$

$\sigma$ is a self-avoiding path from $\mathbf{0}$ to $[N v]$ which successively

$$
\text { visits the vertices } w(0), w(1), \ldots, w(r) \text {. }
$$

Let $\sigma=\sigma(0), \sigma(1), \ldots, \sigma(m)$ and $w(j)=\sigma(s(j))$ with $s(0)=0<s(1)<$ $\cdots<s(r)$. Define further $w(r+1)=[N v]$ and $s(r+1)=m$. Then

the piece $(\sigma(s(j)), \sigma(s(j)+1), \ldots, \sigma(s(j+1)-1)$ of $\sigma$ is contained

$$
\text { in the interior of } B(w(j)), 0 \leqq j \leqq r \text {. }
$$

For $j=r$ we even require that $\sigma(s(r+1))=w(r+1)=[N v]$ lies in the interior of $B(w(r))$. For any path $\pi$ on $\mathbb{Z}^{d}$ denote its length (that is, the number of edges in $\pi$ ) by $|\pi|$. Then

$$
m=|\sigma| \leqq N(\alpha-\delta)
$$

All edges of $\sigma$ except possibly those in $\cup_{j \in \mathcal{S}} B(w(j))$ are white.

$$
|\mathcal{S}|:=\text { cardinality of } \mathcal{S} \leqq \eta N .
$$

We claim that (16) and (20) imply that

$$
r \leqq \frac{N \alpha}{M}
$$

To see this, note that the piece of $\sigma$ from $w(j)$ to $(w(j+1)$ contains at least $\|w(j+1)-w(j)\|=M$ edges, for $0 \leqq j \leqq r-1$. This shows that $N(\alpha-\delta) \geqq$ $m=|\sigma| \geqq r M$. Thus (23) holds. 
Next we note that once $w(j)$ with $j<r$ is given, there are at most $K_{1}:=$ $2 d(2 M+1)^{d-1}$ possible choices for $w(j+1)$, because of (16). As a consequence, the number of sequences $w(0), \ldots, w(r)$ and subsequences $\mathcal{S}$ of $\{0, \ldots, r\}$ for which $\mathcal{F}(\mathbf{w}, \mathcal{S})$ can occur is at most $\left[2 K_{1}\right]^{N \alpha / M}$.

\section{Proposition 3.}

$$
\mathcal{E}(v, N, \delta, \eta) \subset \bigcup_{(w, \mathcal{S})} \mathcal{F}(\mathbf{w}, \mathcal{S}),
$$

where $(\mathbf{w}, \mathcal{S})$ runs over the pairs for which $\mathcal{F}(\mathbf{w}, \mathcal{S})$ is possible. (Thus this union contains at most $\left[2 K_{1}\right]^{N \alpha / M}$ elements.)

Proof. Assume that $\mathcal{E}(v, N, \delta, \eta)$ occurs. By definition, there then exists a selfavoiding path $\sigma$ from $\mathbf{0}$ to $[N v]$ with $|\sigma| \leqq N(\alpha-\delta)$ which contains at most $\eta N$ black edges. We now show that we can choose w and $\mathcal{S}$ such that (16)-(22) all hold. To this end take $s(0)=0$ and $w(0)=\sigma(s(0))=\sigma(0)=\mathbf{0}$. Then for $j \geqq 0$ such that $s(j)$ and $w(j)$ have already been determined such that $w(j)=\sigma(s(j))$, take

$$
\begin{aligned}
s(j+1) & =\inf \{k>s(j):\|\sigma(k)-\sigma(s(j))\|=M\} \\
& =\inf \{k>s(j):\|\sigma(k)-w(j)\|=M\} .
\end{aligned}
$$

We stop the process at the first index $r$ such that $\|\sigma(k)-\sigma(s(r))\|<M$ for all $k>s(r)$ and take $s(r+1)=|\sigma|$, so that $w(r+1)=\sigma(|\sigma|)=[N v]$, the endpoint of $\sigma$. With this choice (16)-(19) are immediate. (For (19) note that $\|\sigma(k)-w(j)\|<M$ for $s(j) \leqq k<s(j+1)$, by virtue of (25).) Also (20) holds by assumption. To get (21) and (22) we take

$$
\begin{array}{r}
\mathcal{S}=\{0 \leqq j \leqq r: \text { there is a black edge in the piece } \\
\sigma(s(j)), \sigma(s(j)+1), \ldots \sigma(s(j+1)) \text { of } \sigma\} .
\end{array}
$$

Equation (21) follows, because any black edge of $\sigma$ belongs to exactly one of the pieces $\sigma(s(j)), \sigma(s(j)+1), \ldots \sigma(s(j+1))$ with $0 \leqq j \leqq r$, and this piece is contained in $B(w(j))$ by (19). Since $\sigma$ contains at most $\eta N$ black edges, by assumption, (22) is also immediate.

We next define the events

$$
\mathcal{G}(\mathbf{i})=\mathcal{G}_{M}(\mathbf{i})=\left\{\text { all edges in } B_{M}(\mathbf{i}) \text { are white }\right\}
$$

and

$$
\mathcal{H}(\mathbf{w}, \mathcal{S})=\bigcap_{j \in \mathcal{S}} \mathcal{G}(w(j))
$$

The following proposition is a kind of converse of Proposition 3. Note that $\mathcal{E}(N, v, \delta, 0)$ is the event that there exists a white path of length at most $N(\alpha-\delta)$ from $\mathbf{0}$ to $[N v]$. 
Proposition 4. For each pair $(\mathbf{w}, \mathcal{S})$ for which (17)-(22) hold, it is the case that

$$
\mathcal{F}(\mathbf{w}, \mathcal{S}) \cap \mathcal{H}(\mathbf{w}, \mathcal{S}) \subset \mathcal{E}(v, N, \delta, 0)
$$

Proof. Assume that $\mathcal{F}(\mathbf{w}, \mathcal{S}) \cap \mathcal{H}(\mathbf{w}, \mathcal{S})$ occurs, and let $\sigma$ be a self-avoiding path from 0 to $[N v]$ for which (18)-(22) hold. Let $\sigma=(\sigma(0), \sigma(1), \ldots, \sigma(m))$. Let $\mathcal{S}=\left\{0 \leqq j_{1}<\cdots<j_{t}\right\}$ with $t \leqq \eta N$. Then $\sigma$ runs from $\mathbf{0}$ to $[N v]$ and it contains only white edges. Indeed, by (21) there are no black edges in $\sigma$ except possibly edges in $B(w(j))$ for some $j \in \mathcal{S}$. However, for $j \in \mathcal{S}$ there are no black edges in $B(w(j))$ either, because we assumed that $\mathcal{G}(w(j))$ occurs. Finally, the length of $\sigma$ is at most $N(\alpha-\delta)$, by virtue of (20). Thus $\mathcal{E}(v, N, \delta, 0)$ does indeed occur.

We next compare the probabilities of $\mathcal{F}(\mathbf{w}, \mathcal{S})$ and of $\mathcal{F}(\mathbf{w}, \mathcal{S}) \cap \mathcal{H}(\mathbf{w}, \mathcal{S})$.

\section{Proposition 5. Let}

$$
K_{2}=d 2 M(2 M+1)^{d-1}
$$

Then, for each pair $(\mathbf{w}, \mathcal{S})$ for which (17)-(22) hold, it is the case that

$$
P\{\mathcal{F}(\mathbf{w}, \mathcal{S})\} \leqq p^{-K_{2} \eta N} P\{\mathcal{F}(\mathbf{w}, \mathcal{S}) \cap \mathcal{H}(\mathbf{w}, \mathcal{S})\} .
$$

Proof. The number of edges in any $B_{M}(\mathbf{i})$ equals $K_{2}$. Therefore,

$$
\text { the number of edges in } \cup_{j \in \mathcal{S}} B(w(j)) \leqq \eta N K_{2} \text {. }
$$

Now notice that, for given $(\mathbf{w}, \mathcal{S})$, the event $\mathcal{F}(\mathbf{w}, \mathcal{S})$ is increasing (in the white edges). Therefore, if $\mathcal{F}(\mathbf{w}, \mathcal{S})$ occurs in some sample point, then $\mathcal{F}(\mathbf{w}, \mathcal{S})$ still occurs if we change any collection of edges to white. Now let $\mathcal{B}$ be an increasing event $\mathcal{B}$ and fix the configuration outside a given edge $e$. Then either $\mathcal{B}$ occurs only if $e$ is white, or $\mathcal{B}$ occurs no matter what the color of $e$ is. In the former case, the conditional probability of $\mathcal{B}$ (given the configuration outside $e$ ) is the same as the conditional probability of $\mathcal{B} \cap\{e$ is white $\}$. In the latter case, the conditional probability of $\mathcal{B}$ equals 1 and the conditional probability of $\mathcal{B} \cap\{e$ is white $\}$ equals $p$. By averaging over the configuration outside $e$ this shows that

$$
P\{\mathcal{B}\} \leqq \frac{1}{p} P\{\mathcal{B}, e \text { is white }\} .
$$

The inequality (28) now follows by successively applying this argument to each edge in $\cup_{j \in \mathcal{S}} B(w(j))$ and taking (29) into account.

The following final proposition proves Lemma 1.

Proposition 6. For $\delta>0$ there exists an $\eta>0$ and a $K_{3}>0$ such that for all large $N$

$$
P\left\{\mathcal{E}(v, N, \delta, \eta\} \leqq \exp \left[-K_{3} N\right] .\right.
$$


Proof. Fix $\delta>0$. By Proposition 2 there exists some constant $K_{4}=K_{4}(\delta)>0$ such that

$$
P\left\{\mathcal{E}(v, N, \delta, 0\} \leqq \exp \left[-K_{4} N\right]\right.
$$

for all large $N$. Proposition 4 then tells us that for each permissible $(\mathbf{w}, \mathcal{S})$ it also holds

$$
P\{\mathcal{F}(\mathbf{w}, \mathcal{S}) \cap \mathcal{H}(\mathbf{w}, \mathcal{S})\} \leqq \exp \left[-K_{4} N\right]
$$

for all large $N$. Then, by Proposition 5,

$$
P\{\mathcal{F}(\mathbf{w}, \mathcal{S})\} \leqq p^{-K_{2} \eta N} \exp \left[-K_{4} N\right]
$$

for all large $N$. Since $K_{2}$ depends on $M$ only, we can for given $\delta$ and $M$ choose $\eta$ so small that

$$
K_{2} \eta \log \frac{1}{p} \leqq \frac{K_{4}}{2}
$$

For such a choice we even have

$$
P\{\mathcal{F}(\mathbf{w}, \mathcal{S})\} \leqq \exp \left[-K_{4} N / 2\right]
$$

for all large $N$. Note that these estimates are uniform in the choice of $(\mathbf{w}, \mathcal{S})$ which satisfy (15)-(22). By Proposition 3 this finally gives

$$
\begin{aligned}
P\{\mathcal{E}(v, N, \delta, \eta)\} & \leqq \sum_{(\mathbf{w}, \mathcal{S})} \exp \left[-K_{4} N / 2\right] \leqq\left[2 K_{1}\right]^{N \alpha / M} \exp \left[-K_{4} N / 2\right] \\
& =\left[4 d(2 M+1)^{d-1}\right]^{N \alpha / M} \exp \left[-K_{2} N / 2\right]
\end{aligned}
$$

for large $N$. We can therefore first choose $M$ so large that

$$
\left[4 d(2 M+1)^{d-1}\right]^{\alpha / M} \leqq \exp \left[K_{2} / 4\right]
$$

and then $\eta$ so small that (31) holds. Result (30) with $K_{3}=K_{4} / 4$ then follows.

Acknowledgments We gratefully acknowledge the support of GNAMPA through the project Homogenization techniques and asymptotic methods for problems with multiple scales coordinated by V. Chiadò Piat. A. Braides' work is part of the Marie Curie Research Training Network MRTN-CT-2004-505226 "Multi-scale modelling and characterisation for phase transformations in advanced materials" (MULTIMAT). The work of ANDREY PIATNITSKI has also been supported in part by RFBR, grants 00-01-22000 and 02-01-00868. 


\section{References}

1. Alicandro, R., Braides, A., Cicalese, M.: Continuum limits of discrete thin films with superlinear growth densities. Calc. Var. Partial Diff. Eq. (to appear)

2. Alicandro, R., Cicalese, M.: A general integral representation result for continuum limits of discrete energies with superlinear growth. SIAM J. Math. Anal. 36, 1-37 (2004)

3. Alicandro, R., Focardi, M., Gelli, M.S.: Finite-difference approximation of energies in fracture mechanics. Ann. Scuola Norm. Pisa (IV) 29, 671-709 (2000)

4. Ambrosio, L., Fusco, N., Pallara, D.: Functions of Bounded Variation and Free Discontinuity Problems. Oxford University Press, Oxford 2000

5. Blake, A., Zisserman, A.: Visual Reconstruction. MIT Press, Cambridge, 1987

6. Braides, A.: Approximation of Free-Discontinuity Problems. Lecture Notes in Math., vol. 1694. Springer, Berlin, 1998

7. Braides, A.: $\Gamma$-convergence for Beginners. Oxford University Press, Oxford 2002

8. Braides, A., Chiadò Piat, V.: Integral representation results for functionals defined in $\operatorname{SBV}\left(\Omega ; \mathbb{R}^{\mathrm{m}}\right)$. J. Math. Pures Appl. 75, 595-626 (1996)

9. Braides, A., Dal Maso, G., Garroni, A.: Variational formulation of softening phenomena in fracture mechanics: the one-dimensional case. Arch. Ration. Mech. Anal. 146, 23-58 (1999)

10. Braides, A., FrancFort, G.: Bounds on the effective behavior of a square conducting lattice. R. Soc. Lond. Proc. Ser. A Math. Phys. Eng. Sci. 460, 1755-1769 (2004)

11. Braides, A., Gelli, M.S.: The passage from discrete to continuous variational problems: a nonlinear homogenization process. Nonlinear Homogenization and its Applications to Composites, Polycrystals and Smart Materials (Eds. Ponte Castaneda P., Telega J.J. and Gambin B.) Kluwer, Dordrecht, pp. 45-63, 2004

12. Braides, A., Solci, M., Vitali, E.: A derivation of linear elastic energies from pairinteraction atomistic systems. Netw. Heterog. Media 2, 551-567 (2007)

13. Сhambolle, A.: Un théorème de $\Gamma$-convergence pour la segmentation des signaux. C. R. Acad. Sci., Paris, Ser. I 314, 191-196 (1992)

14. Сhambolle, A.: Image segmentation by variational methods: Mumford and Shah functional and the discrete approximations. SIAM J. Appl. Math. 55, 827-863 (1995)

15. Cortesani, G., Toader, R.: A density result in SBV with respect to non-isotropic energies. Nonlin. Anal. 38, 585-604 (1999)

16. Dal Maso, G.: An Introduction to $\Gamma$-convergence. Birkhäuser, Boston 1993

17. De Giorgi, E., Ambrosio, L.: Un nuovo funzionale del calcolo delle variazioni. Atti Accad. Naz. Lincei Rend. Cl. Sci. Fis. Mat. Nat. 82, 199-210 (1988)

18. Friesecke, G., Theil, F.: Validity and failure of the Cauchy-Born hypothesis in a two-dimensional mass-spring lattice. J. Nonlin. Sci. 12, 445-478 (2002)

19. Garet, O., Marchand, R.: Asymptotic shape for the chemical distance and firstpassage percolation on the infinite Bernoulli cluster. ESAIM Probab. Stat. 8, 169-199 (2004)

20. Garet, O., Marchand, R.: Large deviations for the chemical distance in supercritical Bernoulli percolation. Ann. Probab. 35, 833-866 (2007)

21. Griffith, A.A., The phenomenon of rupture and flow in solids. Phil. Trans. R. Soc. Lond. A 221, 163-198 (1920)

22. Grimmett, G.: Percolation, 2nd edn. Grundlehren der Mathematischen Wissenschaften, vol. 321. Springer, Berlin, 1999

23. Grimmett, G., Kesten, H.: First-passage percolation, network flows and electrical resistances. Z. Wahrsch. Verw. Geb. 66, 335-366 (1984)

24. Kesten, H.: Percolation theory for mathematicians. Progress in Probability and Statistics, vol. 2. Birkhäuser, Boston 1982

25. Kesten, H.: Aspects of first-passage percolation. Ècole d'été de probabilités de SaintFleur, XIV-1984. Lecture Notes in Math., vol. 180 (Ed. Hennequin P.L.) Springer, Heidelberg, pp. 125-262, 1986

26. Mumford, D., Shah, J.: Optimal approximation by piecewise smooth functions and associated variational problems. Commun. Pure Appl. Math. 17, 577-685 (1989) 
27. Zhang, Y., Zhang, Y.C.: A limit theorem for $N_{0 n} / n$ in first passage percolation. Ann. Probab. 12, 1068-1076 (1984)

Dipartimento di Matematica,

Università di Roma 'Tor Vergata', via della Ricerca Scientifica, 00133 Rome, Italy.

e-mail: braides@mat.uniroma2.it

$$
\text { and }
$$

Department of Mathematics, Narvik University College HiN, Postbox 385, 8505 Narvik, Norway.

$$
\text { and }
$$

P.N. Lebedev Physical Institute, RAS 53 Leninski prospect, Moscow 119991, Russia. e-mail: andrey@sci.lebedev.ru

(Received January 30, 2006 / Accepted September 29, 2007)

(C) Springer-Verlag (2008) 